\title{
APPLICATION OF FUZZY MORPHOLOGHY FOR IMAGE ANALYSIS
}

\author{
Sqn Ldr Atif Bin Mansoor ${ }^{1}$, Flt Lt Adil Khan ${ }^{2}$ and Lt Col Dr Shoab A Khan ${ }^{3}$
}

\begin{abstract}
The word morphology represents a branch of biology, which deals with the form and structure of animals and plants. Mathematical morphology, in the same context, is a tool for obtaining image structures that are helpful in identifying region shape, boundaries, skeletons etc. It is completely based on crisp set theory. Fuzzy set, in contrast to crisp set, is a set whose boundary is not sharp, or precise.

This paper proposes a structure based separation approach of image objects using fuzzy extension of morphology. Using logical operators in fuzzy context, we apply alpha cut morphological processing for cleaning, background subtraction and edge detection. The proposed scheme can be used for many applications like segmentation, thinning and object recognition. We particularly employ fuzzy morphology to aerial images to identify various structures/objects like runways etc. In battlefield, it facilitates to identify friendly and enemy forces and structures. The identification of structural objects is also important in surveys of areas where geographic changes have occurred due to natural disasters.
\end{abstract}

\section{KEY WORDS}

Mathematical Morphology, Fuzzy Morphology, a-Morphology, Dilation, Erosion

\footnotetext{
${ }^{1}$ College of Aeronautical Engg, Pakistan Air Force Academy, Risalpur, Pakistan.

Email : Atif-cae@nust.edu.pk

${ }^{2}$ College of Aeronautical Engg, Pakistan Air Force Academy, Risalpur, Pakistan.Email : adil53@yahoo.com

${ }^{3}$ College of Electrical \& Mechanical Engg, National University of Science \& Technology, Pakistan

Email : kshoab@yahoo.com
} 


\section{Introduction}

In this paper, we illustrate the use of fuzzy morphology for image segmentation and enhancement. We use alpha cut fuzzy morphology to extract the boundary of the desired target by defining a measure of connected components. The extracted edges are superimposed on the original image to increase contrast. The proposed method can be employed as pre or post processing steps in various applications like object detection and identification in military, medical or geographical domains.

\section{Mathematical Morphology}

The word morphology represents a branch of biology, which deals with the form and structure of animals and plants. Mathematical morphology, in the same context, is a tool for obtaining image structures that are helpful in identifying region shape, boundaries, skeletons etc. It is a mathematical framework for the analysis of spatial structures and is completely based on set theory. Thus, sets in mathematical morphology represent objects in an image. Morphological transformations of an image are acquired under the application of local operators, called structuring elements. Shape of structuring element is chosen according to a priori information about the geometry of the interested image structures, and consequently the image is separated into specific image regions due to their structural diversity. The primitive morphological operations are dilation and erosion, while other important morphological operations are opening and closing. Further morphological operations can be obtained from the basic operations of dilation and erosion [1].

\section{Fuzzy Set Theory}

There is always vagueness of things in life. Fuzzy logic is mainly responsible for representation and processing of vague data (ill defined, fuzzy). Fuzzy set theory is the extension of conventional (crisp) set theory. It handles the concept of partial truth, values between 1 (completely true) and 0 (completely false). Fuzzy set is a set whose boundary is not sharp, or precise. This concept contrasts with classical concept of a set, called a crisp set, whose boundary is required to be precise.

\section{Fuzzy Image Processing}

Fuzzy image processing has three main stages: image fuzzification, modification of membership values, and, if necessary, image defuzzification (Figure 1). [2] 
The fuzzification and defuzzification steps are due to the fact that we do not possess fuzzy hardware. Therefore, the coding of image data (fuzzification) and decoding of the results (defuzzification) are steps that make possible to process images with fuzzy techniques.

\section{Image Analysis Using Fuzzy Morphology}

There has been considerable interest in fuzzy image processing [3], [4]. The advantage of fuzzy logic in image processing results from the possibility to overcome the crisp nature of pattern descriptions. A gray scale image is a fuzzy set in the sense that it is a fuzzy version of a binary image, or that a gray value represents the degree to which a pixel belongs to the image foreground. Similarly, for detection tasks it is always important to extract a spatial connected part of the image as foreground, and to remove all remaining parts of the image as background.

In our approach, we applied top-hat transformation on image using fuzzy dilation and erosion to enhance details, even in presence of shading. The morphological top-hat transformation of an image, denoted $h$, is defined as [1] :

$h=f-\left(f^{\circ} b\right)$

where ' $f$ ' is the input image and ' $b$ ' is the structuring element function. The opening of ' $f$ ' by ' $b$ ' is the erosion of ' $f$ ' by ' $b$ ', followed by a dilation of the result by ' $b$ '. In mathematical terms

$f^{\circ} b=(f \oplus b) \Theta b$

We used an adaptive diamond shaped structuring element of radius 5 , and calculated the super minima. The fuzzification process was done through the alpha cuts of the fuzzy membership functions. a-cuts give a very convenient way for linking fuzzy concepts and crisp concepts [5]. Given a fuzzy set $\mu(x)$, where $x$ is an element of the so-called universe of discourse $X$ and assigning membership degrees from the interval $[0,1]$ to each element of $X$, then for $0 \leq \alpha \leq 1$, the $\alpha$-cut of $\mu(x)$ is the set of all $x \in X$ with membership degree at least as large as $\alpha$ :

$\mu_{\alpha}=\{x \mid \mu(x) \geq \alpha\}$

By using a -cuts, all standard operations of fuzzy sets can be derived from their crisp counterparts. The fuzzy dilation was done as given in [7], [8]. 
$(\mathrm{g} \oplus \mu)(\mathrm{x})=\sup _{\mathrm{y} \in \mathrm{X}} \min [\mathrm{g}(\mathrm{x}-\mathrm{y}), \mu(\mathrm{y})]$

The corresponding fuzzy erosion was done as:

$(\mathrm{g} \ominus \mu)(\mathrm{x})=\inf _{\mathrm{y} \in X} \max \left[g_{\max }-\mathrm{g}(\mathrm{x}-\mathrm{y}), \mu(\mathrm{y})\right]$

The challenge was to find suitable fuzzy structuring element. We found out that for structuring element the alpha chosen as complement of highest frequency in image histogram is the most appropriated choice. After completing the top hat filtering, gray scale image was converted into binary by selecting the optimum threshold level using the Otsu method [6]. We employed thinning by hit or miss transform for shape detection. The thinned set is converted to m-connectivity to eliminate multiple paths. Small objects are removed from the image by keeping only connected pixels. The image can be altered by varying the quantity of connected pixels. The resultant image is added to original image offering improved visibility of the target object.

\section{Experimental Results}

For our analysis, we used the aerial images. Aerial images are used to identify various structures/objects in peace and war. In battlefield, it facilitates to identify friendly and enemy forces and structures. The aerial image, shown in figure 2 was used. The aim was to detect the runway using fuzzy morphology. The result of the application of our method resulted in the image shown in figure 3 . We selected criteria of maintaining thousand connected pixels to extract the runway. Lowering the number of connected pixels give us even the secondary and tertiary tracks. Figure 4 gives an enhanced version of our original image by adding the segmented runway object into original image.

\section{Conclusion}

We present an image segmentation utilising the alpha cut fuzzy morphology approach to extract desired objects from an image. The challenge was to find suitable fuzzy structuring element that was found by selecting alpha equal to complement of highest frequency intensity levels in image histogram. Satisfactory results were achieved using our method. The approach can be applied in various applications related to military, geographical surveys, objects identification etc. 


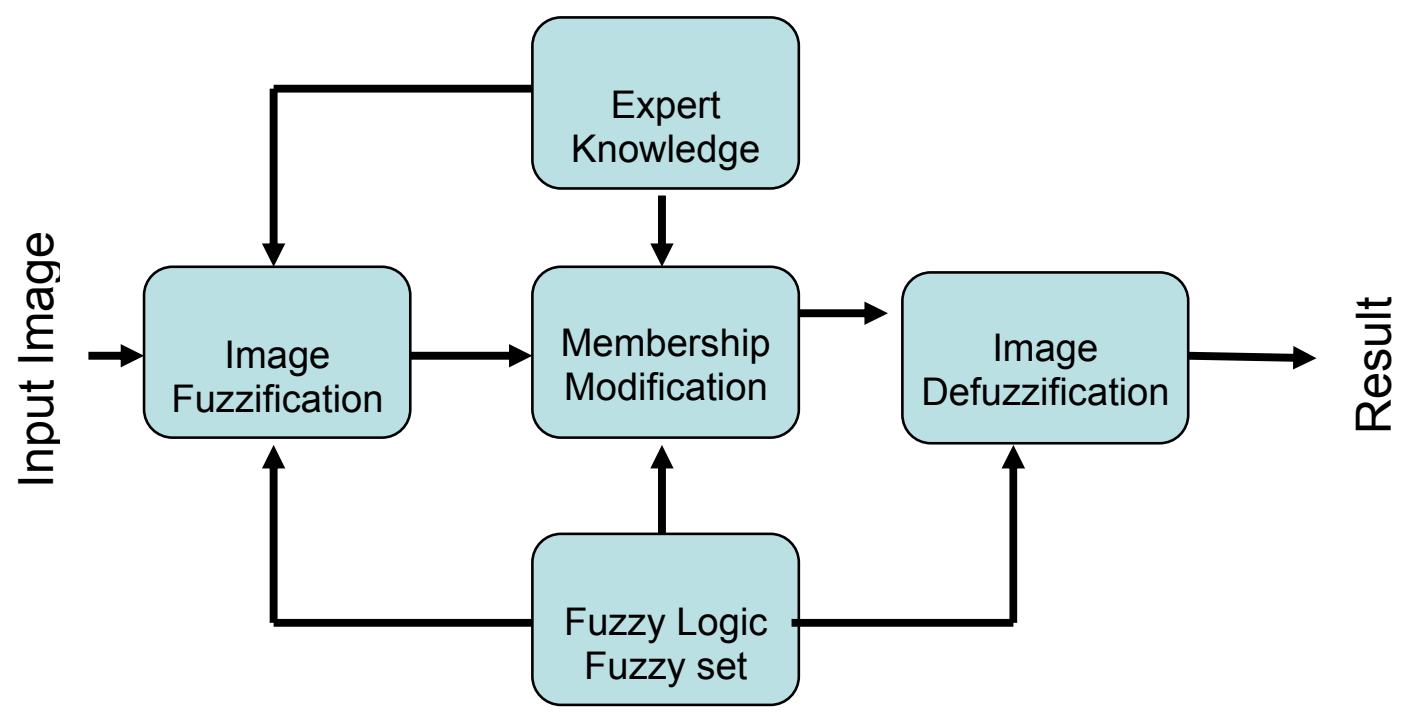

Figure 1 : FUZZY IMAGE PROCESSING STAGES

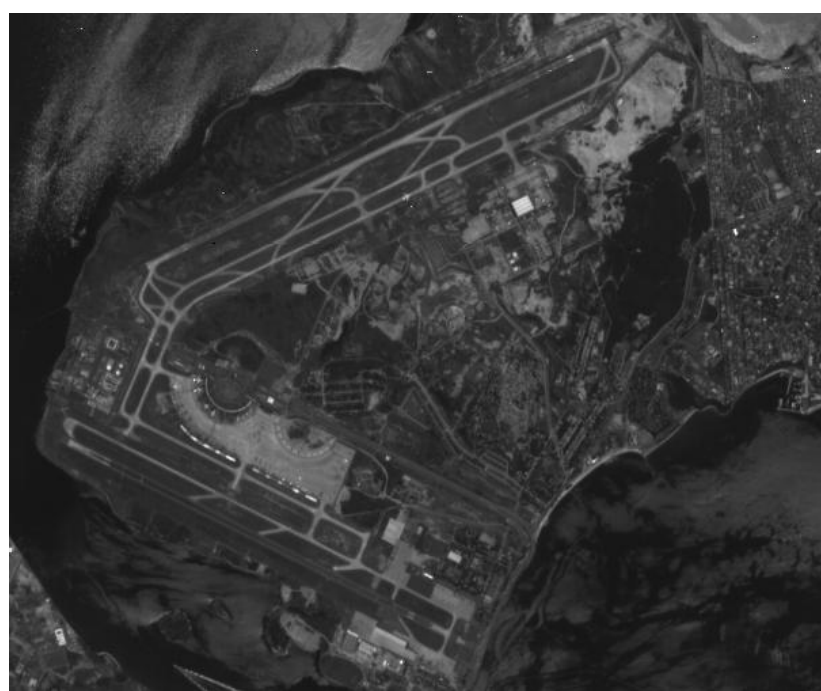

Figure 2 : Original Aerial Image 


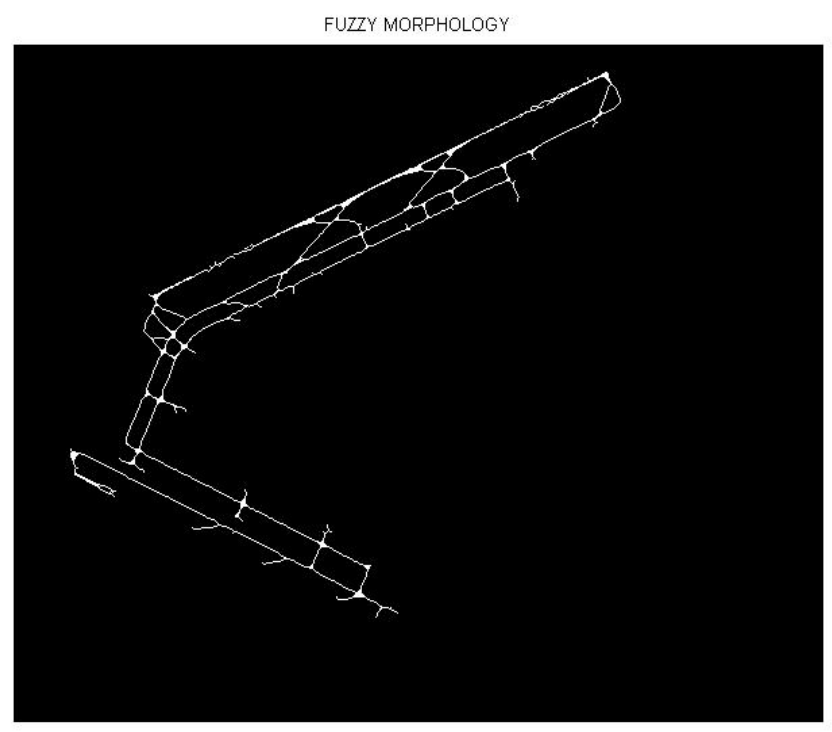

Figure 2 : Runway detection using Fuzzy Morphology

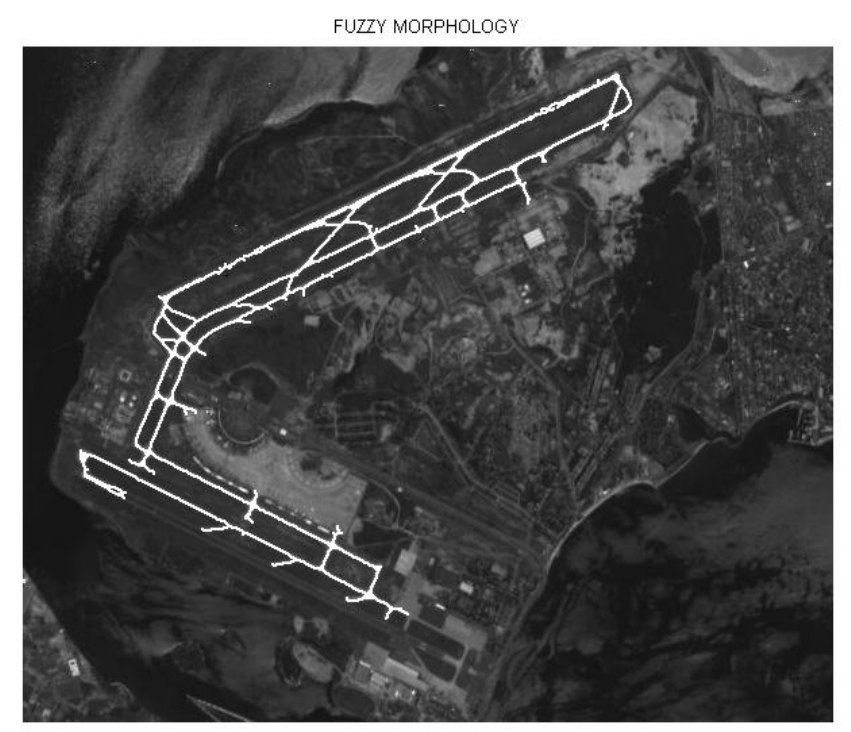

Figure 3 : Enhanced Aerial Image

\section{REFERENCES}

1. Gonzalez, Digital Image Processing, Prentice Hall, (2005).

2. Tizhoosh, Fuzzy Image Processing, Springer, (1997).

3. Wirth, Nikitenko, "Application of fuzzy morphology to contrast enhancement”, NAFIPS, (2005) 
4. Deng, Heijman, "Grey scale Morphology based on Fuzzy logic", J. Math. Imaging and Vision, 16, 155-171, (2002).

5. Koeppen, Franke, "A tutorial on fuzzy morphology", Frauenhofer IPK, Berlin

6. Otsu, N., "A Threshold Selection Method from Gray-Level Histograms," IEEE Transactions on Systems, Man, and Cybernetics, Vol. 9, No. 1, pp. 62-66, (1979).

7. Bloch, H. Ma itre, Fuzzy mathematical morphologies: A comparative study, Pattern Recognition, 28, pp. 1341-1387, (1995).

8. A. Kaufmann, M.M. Gupta, Fuzzy Mathematical Models in Engineering and Management Science, North-Holand, Amsterdam, (1988). 